\title{
Researches regarding cutting tool condition monitoring
}

\author{
Marinela Inţă $^{1}{ }^{, *}$, Achim Muntean ${ }^{1}$, and Sorin-Mihai Croitoru ${ }^{2}$ \\ ${ }^{1}$ Lucian Blaga University of Sibiu, Industrial Engineering and Management Department, Victoriei, \\ 10, 550025, Sibiu, Romania \\ ${ }^{2}$ Politehnica University of Bucharest, IMST Faculty, Spl. Independentei, 313, Bucharest, Romania
}

\begin{abstract}
The paper main purpose is monitoring of tool wear in metal cutting using neural networks due to their ability of learning and adapting their self, based on experiments. Monitoring the cutting process is difficult to perform on-line because of the complexity of tool wear process, which is the most important parameter that defines the tool state at a certain moment. Most of the researches appraise the tool wear by indirect factors such as forces, consumed power, vibrations or the surface quality. In this case, it is important to combine many factors for increasing the accuracy of tool wear prediction and establish the admissible size of wear. For this, paper both the theoretical data obtained from FEM analyze and experimental ones are used and compared in order to appreciate the reliability of the results.
\end{abstract}

\section{Introduction}

The most updated techniques for intelligent tool condition monitoring which are based on information from different sensors integrated with a neural network [1]. The artificial neural networks $(\mathrm{NN})$ consist of many massive interconnections of rather simple neurons simulating the biological nervous system. These networks are also referred to as parallel distributed processing. Based on the modification of weights, which came from the experience, the network can learn from its past experience, so as to simulate the human brain. So, we can say these type of networks is intelligent, [2].

In any machining process, the survey of the tool state plays a central role in the process diagnosis system. During this process, the tool also loses the capacity of cutting, which is referred as tool wear. The size of the tool wear is the common way to evaluate the tool state.

The monitoring of gradual wear requires the development of sensitive, accurate, and reliable devices. On-line monitoring and compensation of the tool wear would be of a great help to avoid the increase in cutting force [3] loss of accuracy, deterioration in surface finish, increase in cutting temperature and increase in vibration due to tool wear.

\footnotetext{
*Corresponding author: marinela.inta@ulbsibiu.ro
} 
The main types of monitoring the tool wear are direct and indirect methods. Although, both methods use different sensors to monitor the tool wear, but they differ in some aspects.

In the direct method [4], sensors directly measures the tool wear, such as optical scanning technique, electrical resistance, radioactive technique, measurement of tool geometry, change in work piece size, and analysis of tool wear particles in the chips. The difficulties of direct methods lead to the indirect measuring techniques to measure accessible process variables (machine tool vibrations, acoustic emission, temperature, noise etc.), which are related to tool wear and correlating the changes in these parameters to the change in the tool wear. The static methods use some static characteristics of the monitoring signal such as the mean the RMS [5]. Unfortunately, static methods are often too sensitive to the variation of the cutting conditions and not suitable for on-line monitoring. Therefore, the dynamic methods are developed, which use the dynamic characteristics of the monitoring signal to identify and predict the tool condition.

The majority of the techniques discussed [6] above for tool wear sensing are limited due to influence of external disturbances unrelated to tool wear, and inability of instrumentation to operate reliably in the immediate vicinity of the cutting process. Investigations have been carried out by several researchers on various tool wear sensors based on radioactive isotopes. The input settings of the process are selected by evaluating, on-line, a set of feasible alternatives with respect to several criteria. Relevant performance measures such as process cost and production rate can be directly influenced in this approach by establishing appropriate definitions for the decision criteria.

\section{Monitoring of cutting tool state}

Monitoring of the machining process has an important role in avoiding to long idle time of the machine or preventing undesired events like loss of accuracy, excessive tool wear or even the failure of the cutting tool.

The researches looking the conception of any tool state monitoring systems is continuing for a while and even if the technology of sensors and computers has evolved, the use in industry is poor, [7]. As [1] "suggested", despite more a decade of intensive scientific research, the development of the monitoring systems of the tool wear is still an attempt in course of development", even if a big variety of monitoring techniques have been reported and many publications presented satisfactory performances. Thus, the success of a monitoring system of the tool state depends in a great measure upon the capacity of the system to identify any anomaly and to answer on-line with a corresponding action.

Measuring and analyze on purpose of diagnosis and monitoring assumes the conception of certain hierarchic structures in a modular construction so that each module has to solve the necessary input data for the next modules based on the data from previous ones. This could be achieve based on information for each module and by inclusion in it of a specialized monitoring function depending on the possible defect to appear.

For realizing an on-line tool condition monitoring system, we assume that:

- Normal function of the process depends directly on the tool condition; bad function means an altered condition of the tool, that could be detected by the system;

- After detecting of an aberrant signal, the system must analyze and decide in accordance with imposed conditions. The answer is send to the machine tool system for execution of the received order;

- Uses of artificial intelligence by means of neural networks/ fuzzy logic increase the accuracy and reliability of the system.

From the earlier researches, one can notice that the main parameters used to monitor the tool wear are the cutting forces, vibrations, acoustic emission and the power consumed [8]. 
Because all these parameters are indirect indicators of tool wear the use of single or only two parameters causes a bad accuracy and reliability of the system. In a few cases, also the temperature of the tool is considered, but due to difficulties encountered at the practical measure of that, it is not practically useful.

This paper analyzes the possibility of using temperature of the cutting tool to be one of the three parameters to indicate the state of wear of the cutting tool: main cutting force, temperature and acoustic emission.

The researchers are focused on introducing the temperature as the third monitored parameter of the wear, because all analytical models for the tool wear contain the temperature as main parameters and everybody assumes that the main causes of toll wear are mechanical and thermal loads. Also, the prediction of tool wear has the form of temperature field in FEM analyze.

\section{Tool wear simulation with FEM}

Concerning the topic of the present paper the introduction of the FEM in the field of deformation in elastoplastic media made possible the use of this method in the metal cutting process. For establishing a theoretical model for the cutting temperature and forces, Deform 2D Machining software is used, for modeling and simulating of the cutting process, using the assumptions of orthogonal cutting.

The software can simulate the cutting process using different cutting parameters like cutting speed, feed and depth of cut for computing the cutting forces, temperature of cutting zone, stress and strain state in chip and work piece, appreciation of chip form and estimation of the tool wear and tool life.

The data referring to the force, temperature and tool wear prediction (based on the Usui model) obtained from the simulation with FEM are used for preliminary training of the NN. In this way, we can obtain a theoretical function of the tool state, which links the tool wear with the three parameters monitored.

Based on Usui Model (eq. 1) a program for solving the tool wear was developed and the theoretical values of wear were calculated:

$$
\frac{d w}{d t}=C_{1} \cdot \sigma \cdot v_{s} \cdot e^{\left(\frac{-C_{2}}{T}\right)}
$$

where $\frac{d w}{d t}-$ wear rate;

$\mathrm{C} 1, \mathrm{C} 2$ - coefficients depending on tool and piece materials

$v_{s}$ - chip shear velocity, $[\mathrm{m} / \mathrm{min}]$,

$\mathrm{T}$ - cutting temperature

$\sigma$ - normal pressure

In order to obtain some input data for the algorithm of calculus of tool wear, referring at load, forces, temperature a series of simulations were performed in DEFORM 2D software.

For the process of simulation there was used a tool made of hard metal (WC), covered with a layer of 5 microns of titanium carbo-nitrid (TiCN), and for the work piece material a C45 steel annealed and a $50 \mathrm{~mm}$ in diameter specimen. Cutting parameters are presented in table 1 together with working conditions that are introduced in pre - processor.

Simulation with FEM is made for determine the size of forces in order to obtain a model of forces for the cutting process, taking into consideration the tool and process parameters and also to determine the temperature of the tool. In case of a new one and considering different degrees of the tool wear, making the assumption that the temperature increase 
with the degree of wear due to the change in tool geometry and increase of the friction forces.

Table 1. Input data for simulation

\begin{tabular}{|c|c|c|c|c|c|}
\hline Nr. & Tool geometry & & $\begin{array}{c}\text { Feed, } \\
{[\mathrm{mm} / \text { rot }]}\end{array}$ & $\begin{array}{c}\text { Depth of cut, } \\
{[\mathrm{mm}]}\end{array}$ & $\begin{array}{c}\text { Cutting speed } \\
{[\mathrm{m} / \mathrm{min}]}\end{array}$ \\
\hline 1. & & $\alpha=6^{0}$ & 0,07 & 0,5 & 52 \\
\hline 2. & & 50 & 0,14 & 1,0 & 100 \\
\hline 3. & & $r_{\varepsilon}=0,4$ & 0,28 & 1,5 & 200 \\
\hline 4. & & $\alpha=6^{0}$ & 0,07 & 0,5 & 52 \\
\hline 5. & & $\gamma=-5^{0}$ & 0,14 & 1,0 & 100 \\
\hline 6. & $t^{\alpha}$ & $r_{\varepsilon}=0,4$ & 0,28 & 1,5 & 200 \\
\hline 7. & & $\alpha=6^{0}$ & 0,07 & 0,5 & 52 \\
\hline 8. & & $\gamma=5^{0} ; w=0,2$ & 0,14 & 1,0 & 100 \\
\hline 9. & $w^{i}$ & $\gamma_{f}=-10^{0}$ & 0,28 & 1,5 & 200 \\
\hline
\end{tabular}

Consequently, to the run of the program of finite elements used for the simulation of the turning process the following values for output parameters were obtained:

- the temperature distribution field in the cutting zone (figure 1);

- the isotherms of temperature field in the cutting tool, very useful for the estimation of the tool wear;

- plane stress state in work piece and chip with effective stress;

- the load on $\mathrm{X}$ and $\mathrm{Y}$ for the chip elements in $\mathrm{N}$.

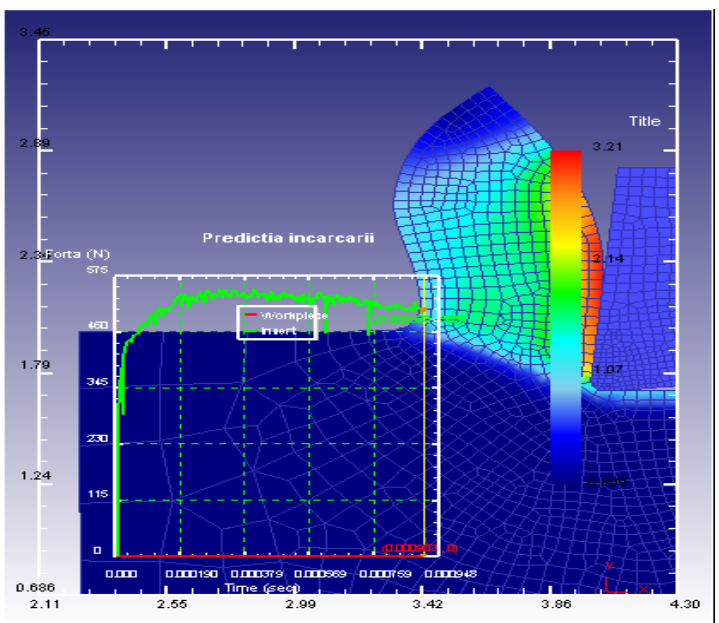

Fig. 1. Evolution of temperature in the turning process (no. 3 from table 1)

With data from FEM simulation, a software product named "calcul_uzura.m" in Matlab software. After running, the program and considering a real cutting time data were obtained and presented in table 2 . 
Table 2. Calculated data (theoretical values)

\begin{tabular}{|l|c|c|c|c|c|}
\hline $\begin{array}{c}\text { Temperature } \\
{[\mathbf{0 C}]}\end{array}$ & $\begin{array}{c}\text { Force } \mathbf{F}_{\mathbf{y}} \\
{[\mathbf{N}]}\end{array}$ & $\begin{array}{c}\text { Wear } \\
{[\mathbf{m m}]}\end{array}$ & $\begin{array}{c}\text { Temperature } \\
{[\mathbf{0 C}]}\end{array}$ & $\begin{array}{c}\text { Force } \mathbf{F}_{\mathbf{y}} \\
{[\mathbf{N}]}\end{array}$ & $\begin{array}{c}\text { Wear } \\
{[\mathbf{m m}]}\end{array}$ \\
\hline 20.00 & 0.00 & 0.00 & 298.00 & 93.82 & 0.50 \\
\hline 111.00 & 9.79 & 0.05 & 302.00 & 109.22 & 0.55 \\
\hline 153.00 & 10.70 & 0.10 & 306.00 & 122.42 & 0.60 \\
\hline 202.00 & 36.82 & 0.15 & 307.00 & 135.62 & 0.65 \\
\hline 227.00 & 56.47 & 0.20 & 315.00 & 148.82 & 0.70 \\
\hline 259.00 & 56.57 & 0.25 & 326.00 & 162.02 & 0.75 \\
\hline 265.00 & 70.45 & 0.30 & 338.00 & 175.22 & 0.80 \\
\hline 278.00 & 81.91 & 0.35 & 349.00 & 186.20 & 0.85 \\
\hline 283.00 & 88.56 & 0.40 & 353.00 & 232.50 & 0.90 \\
\hline 290.00 & 92.10 & 0.45 & 357.00 & 298.76 & 0.95 \\
\hline 298.00 & 93.82 & 0.50 & 359.00 & 338.56 & 1.00 \\
\hline 302.00 & 109.22 & 0.55 & 361.00 & 387.95 & 1.05 \\
\hline 306.00 & 122.42 & 0.60 & 363.00 & 432.99 & 1.10 \\
\hline 307.00 & 135.62 & 0.65 & 367.00 & 547.32 & 1.15 \\
\hline
\end{tabular}

The calculated wear after a certain time cycle could be represented and used in the monitoring system, as the neural network learning curve.

\section{Experimental test}

In order to estimate tool wear by measuring noise experimental tests were carried out on the same machine tool and under the same cutting conditions as in the case of attempts to force, temperature and noise.

Starting from the parameters of data acquisition board National Instruments USB6008, three signals were acquisitioned (F, T and $\mathrm{Z}$ ) with the sampling frequency imposed by hardware, these being the instant values for input in the NN. The three acquisitioned signals are performed using:

- workpiece made of C 45 steel with length $500 \mathrm{~mm}$ having diameter $30 \mathrm{~mm}$ with the following characteristics: $\mathrm{R}_{\mathrm{p} 02}=380 \mathrm{daN} / \mathrm{mm}^{2} ; \mathrm{R}_{\mathrm{m}}=640 \mathrm{daN} / \mathrm{mm}^{2} ; \mathrm{HB} 218$;

- measure of the temperature, $\mathrm{T}$ variation in cutting area, performed by a natural thermocouple tool-work-piece method;

- cutting forces, $\mathrm{F}$ it has been measured by dynamometer in turning;

- measurement of noise, Z was made using Sonometer Quest Technologies 2100.

\subsection{Tool temperature measurement using natural thermocouple}

For measurement and acquisition on-line of cutting tool, temperature there was realized an experimental stand and virtual instrument developed in LabView 8.5 software. It is well known that the main problem encountered in tool temperature using the natural thermocouple is its calibration. 
That problem is caused because of the different materials used for the cutting tool and for the work pieces that are normally different in each operation and product [9]. Using the stand for different natural thermocouple calibration presented in [10] which is table to calibrate simultaneous three types of natural thermocouples with a reference $\mathrm{K}$ type thermocouple we were able to calibrate the used couple of materials. The following calibration equation for the couple P30 - AISI 1045 steel was obtained:

$$
y=9.78+0.382 \cdot x
$$

where:

$\mathrm{y}$ is temperature in $\left[{ }^{\circ} \mathrm{C}\right]$ corresponding to the measured voltage $\mathrm{x}$ in $\mu \mathrm{V}$.

For obtaining the mathematical model of temperature was used a factorial experiment type $3^{2}$, and the following mathematical model was derived:

$$
\begin{aligned}
& T=36.4105+2.0035 \cdot v-1.00776 \cdot f-0.0155 \cdot v \cdot f+1.8452 E-003 \cdot v^{2}+ \\
& +0.010331 \cdot f^{2}
\end{aligned}
$$

Using the ANOVA analysis of the temperature model obtained, we can find influences of each parameter and its concordance. Temperature dependence of the cutting parameters is illustrated by the graphic below (fig.2).

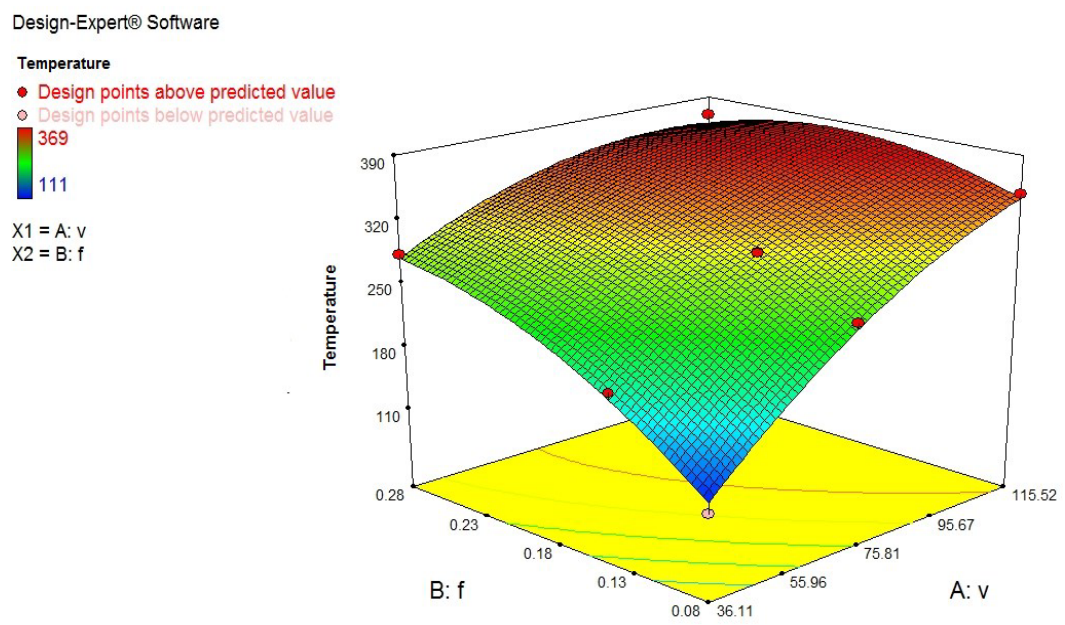

Fig. 2. Interacting factors

We can observe that, the temperature increase more at small feeds that for the greater feeds, where the chip thickness is bigger.

\subsection{Cutting forces measurement}

To determine the influence of the cutting forces on tool wear an inductive dynamometer for measuring cutting forces in turning was used.

To find out a model of the cutting force a full factorial experiment type $3^{3}$ for the three factors and three levels of variation was used, which results in 27 experiments. After data processing with Design of Experiment software the following mathematical model was obtained for the main cutting force: 


$$
\begin{aligned}
& F_{y}=386.76-1908.07 \cdot f-153.33 \cdot a_{p}-7.26 \cdot v+1119.86 \cdot f \cdot a_{p}+23.28 \cdot f \cdot v+ \\
& +4.82 \cdot a_{p} \cdot v
\end{aligned}
$$

The model obtained was analyzed using statistical tests ANOVA and Fisher, which give us the influence of each factor, reliability and diagnostics for each factor. In this case, the degree of confidence used is $95 \%$.

Fisher coefficient calculated for testing the suitability of the proposed model shows that the main cutting force model is completely having factors interactions not considered in the model and influence in a significant way values resulting from measurements.

Table 3. Significance testing factors

\begin{tabular}{|l|l|c|c|l|}
\hline \multicolumn{1}{|c|}{ Source of variation } & Sum of squares & DF & Fcalc & \multicolumn{1}{c|}{ Prob>F } \\
\hline A factor - feed & 83891.70 & 1 & 4.35 & 0.0574 \\
\hline B factor -depth of cut & $4.90 \mathrm{e}^{005}$ & 1 & 25.40 & $0.0002 \Rightarrow$ The most significant \\
\hline C factor - cutting speed & 59155.32 & 1 & 3.06 & 0.1036 \\
& & & & \\
\hline AB factor & 46563.01 & 1 & 2.41 & 0.1444 \\
\hline AC factor & $1.177 \mathrm{e}^{005}$ & 1 & 6.10 & $0.0282 \Rightarrow$ significant \\
\hline BC factor & $1.145 \mathrm{e}^{005}$ & 1 & 5.93 & $0.0300 \Rightarrow$ significant \\
\hline
\end{tabular}

Comparing coefficients calculated by Fisher's test for $\mathrm{F}_{\mathrm{y}}$ component, it appears that, $\mathrm{B}$ factor (depth of cut) is the most significant parameter.

To be able to achieve a prediction of wear (VB) in terms of cutting force, $F_{y}$ we assume that the forces measured during the experiments are distributed equally over the entire area of contact between tool and workpiece. Also in this case it were made a series of measurements on the same cutting conditions, keeping constant cutting conditions and using different wears of inserts. To obtain the mathematical model a factorial experiment of type $3^{3}$ was developed, and after analysis with software Design-Expert the following mathematical model:

$$
\begin{aligned}
& F_{y}=415.80-420.44 \cdot a_{p}-93.24 \cdot f-1204.58 \cdot V B+196.51 \cdot a_{p} \cdot f+ \\
& 1164.97 \cdot a_{p} \cdot V B+3295.42 \cdot f \cdot V B
\end{aligned}
$$

Analyzing the obtained results, the following conclusions can be drawn:

- analysis of cutting force signal (sudden increase or decrease) one can detect a collision, breaking or other undesirable random events;

- for achieving a correlation wear-force a mathematical model was derived both for a new tool and a worn one having a wear rate $\mathrm{VB}=0.25-1.2 \mathrm{~mm}$;

- in the initial experiment the parameter cutting speed was suppressed due to the low influence and replaced with parameter wear, VB that allows to determine a correlation force-wear;

- in the new model the order of influence in the output is VB, $a_{p}, f$ that indicating that force could be used as monitored parameter of the wear process;

\subsection{Noise measurement}

The results of the measurements were processed using a virtual instrument in LabView 8.5 software presented in figure 3. To obtain a clearer signal and for eliminating other factors to intervene in the cutting process a measurement chain of noise band-pass filter was used, which will completely attenuate any signal outside the desired range. 


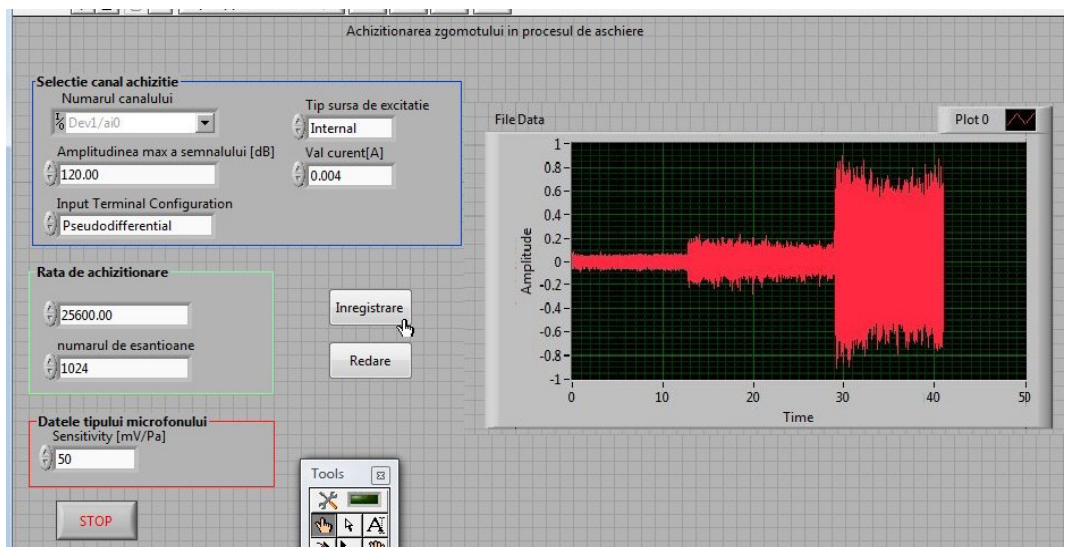

Fig. 3. Virtual instrument for noise analyze

Analyze and interpretation of measurement results for the cases mentioned wear were performed using the software SigView.

The following features were obtained and analyzed:

- noise amplitude (Figure 4),

- analyze FFT (Fast Fourier Transform);

- highest 5 peaks;

- determining the fundamental harmonic signals, etc.

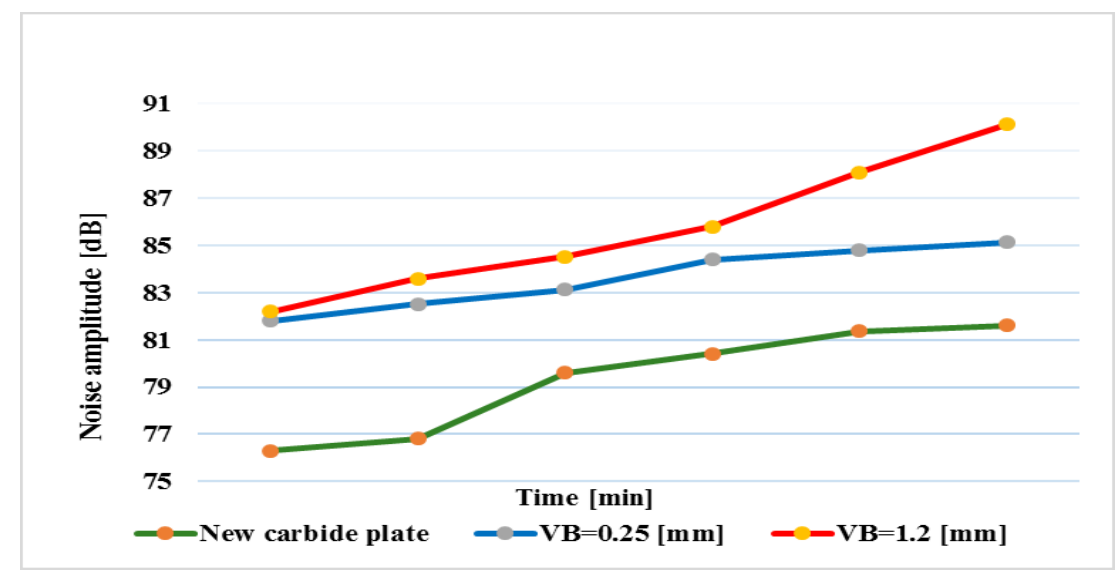

Fig.4.Variation of noise amplitude in time

In order to use the theoretical instead of the experimental data for training the neural network those were compared to the experimental values obtained for forces and temperature with the ones obtained in FEM:

- The temperature difference between the maximum temperature measured and that obtained by finite element simulation is greater (about 30\%), because in experiment we measured the average temperature of tool- chip contact and in FEM it was the maximum tool temperature on a discrete element.

- If we consider the average chip-tool temperature, model accuracy is much better, with less than $3.5 \%$ error. 


\section{The model of neural network (NN) in monitoring}

For better on-line monitoring of machining processes, it is necessary to have the actual process of tool wear. Tool wear can be estimated from the signals of sensors.

The use of NN for monitoring the tool wear involves a series of measures linked with data acquisition, processing and input of the measured values in the NN. The main steps are:

- data acquisition of the signals for forces, temperature (theoretical values) and noise (real values);

- normalization of the data;

- transmission of the data in the input layer of NN.

The Neural network is composed by 3 layers using the inner product DOTPROD of bias functions, the net input function NETSUM and the transfer function TANSIG. The designed network for wear prediction is composed as follows: 3 neurons on the input layer (for force, temperature and noise), 3 neurons in the hidden layer and a neuron for the output (normalized value of the wear). Due to the training back propagation algorithm needs that activation functions must be limited and differentiable a virtual instrument has been realized for the activation function as sigmoid type both for the neurons in the hidden layer and for the output level.

For data normalization, a program in Microsoft Visual Basic was developed. It takes the acquired data in an time interval from Excel Cells in 3 vectors as described in the program code presented below. The result of this processing is that it keeps a constant activity level at the level of a neuron layer. Because of the heterogeneous 3 values of the input data their scales are completely different and that must be eliminated. The normalized data for $\mathrm{NN}$ training having the values between 0 and 1 are presented in figure 6 and the values were used as example for the network training.

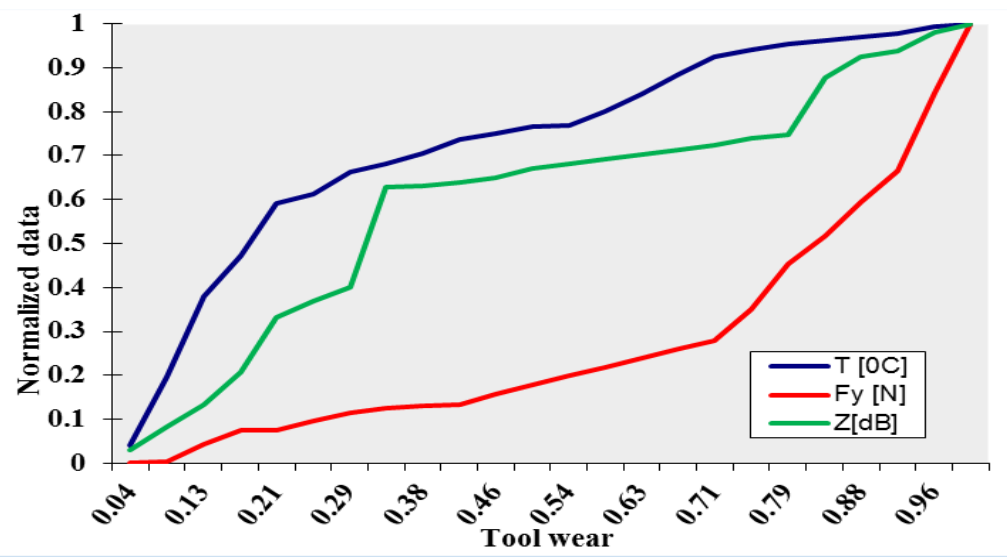

Fig. 6. Template learning using input data

With proposed neural network, model and theoretical data obtained from FEM a NN training was done using theoretical wear function as output and adding the third parameter as measured noise considering that as an improvement of accuracy.

\section{Conclusions}

The main goal of the paper was to show that introducing tool temperature as input factor in neural network training besides forces and noise or vibrations improve accuracy and 
stability of the results. Important researches were performed for improving the method of natural thermocouple for measuring the variation of tool temperature.

Considering both theoretical results and experimental ones, we can obtain an ideal set of input data for neural network training for different sets of tool and workpiece materials. The complex function obtained can be tuned in function of real conditions for each cutting operation, taking into consideration the measured parameters from on line sensors.

The difference between the measured and computed temperature is inside a reasonable limit (approx. 3\% for average temperature) and this can be explained by the fact that the natural thermocouple measures the average temperature of tool-piece interface. Achieved small difference between theoretical and experimental values justify the use of the calculated values instead of experimental and can thus generate databases for preliminary training neural networks

The present paper aims to contribute to the integration of neural network capabilities in the Lab View environment for improving computer-aided control and monitoring of machining processes.

Further researches are proposed for improving the accuracy, reliability and communication between the tool state monitoring and the $\mathrm{NC}$ of the machine tools.

\section{References}

1. B. Stick. Mechanical Systems an Signal Processing, 16(4), 487 (2002)

2. R. J. Schalkoff. Artificial neural networks, (Publisher McGraw- Hill Inc., New York, 1997)

3. A. Salimi, M. Zadshakouyan. Australian Journal of Basic and Applied Sciences, 3(1), 283 (2009)

4. T. Özel, Y. Karpat International Journal of Machine Tools and Manufacture, 45, 467 (2005)

5. R. Allen, H. Shi. International Journal of COMADEM, 4(1), 26 (2001)

6. C. Byrne, D. Dornfeld, I.I.G. Ketteler, W. Konig, R. Teti. CIRP J. Man. Sci. Tec 44, 541 (1995)

7. L. Huajie, J. Dong. Journal of Intelligent Manufacturing, 7 (3), 243 (1996)

8. C. Scheffer, H.,Katz, P.S. Heyns, F. Klocke, Int J Mach Tool Manu, 43, 973 (2003)

9. U. Zuperl, F. Cus, B. Mursec, T.A. Ploj. Journal of Materials Processing Technology, 157, 82 (2004)

10. M. Inţă, A. Muntean, Advanced Materials Research, 1036, 274 (2014) 